\title{
NUMERICAL STUDY OF DISK DRIVE ROTATING FLOW STRUCTURE IN THE CAVITY
}

\author{
Kwan Ouyang ${ }^{1}$, Reui-Kuo Lin ${ }^{2}$, Shun-Feng Tsai ${ }^{3}$ \\ ${ }^{1}$ Associate Professor, Department of Marine Engineering, Taipei College of Maritime Technology, Taipei, Taiwan, \\ Republic of China (R.O.C.) \\ ${ }^{2}$ Assistant Professor, Department of Marine Engineering, Taipei College of Maritime Technology, Taipei, Taiwan, \\ Republic of China (R.O.C.) \\ ${ }^{3}$ Assistant Professor, Department of Marine Engineering, National Taiwan Ocean University, Keelung, Taiwan, \\ Republic of China (R.O.C.)
}

\begin{abstract}
This paper aim in conducting the numerical simulation of laminar flow to explore disk-driven vortical flow structure of a cubical container subjected to a disk rotation on the roof of the container in different Reynolds numbers to observe the flow structure and the reason of vortical flow form. For this study, finite difference method with dispersion-relation-preserving (DRP) scheme is dispersed governing equations space term, but adopt time term with TVD Runge-Kutta method. To add accuracy of numerical, this thesis also uses topology theory to analyze the characteristic of singular point. Three-dimensional vertical flow is observed flow structure and move to condition. The result to obtain Reynolds numbers to increase attracting spiral nodes increasingly approaches the floor of the cavity. We have also depicted the vertical flow structure in terms of cortex cores which provide more details about how change of the Reynolds number
\end{abstract}

Keywords: disk-driven, finite difference method, dispersion-relation-preserving (DRP), Runge-Kutta, topology theory

\section{INTRODUCTION}

Numerical simulation is based on mathematical rules, computer technology, engineering and related disciplines of professional. From the school gate, in recent years, due to advances in computer hardware and software, numerical calculation method improved physical problems for more accurate analytical results. Numerical calculations have been widely used in the field of physics, chemistry, fluid dynamics, atmospheric science, aerospace, and industrial applications such as solid mechanics. Hydrodynamics is part of mechanics and it is mainly Fluid (including gas and liquid) scientific phenomena and related mechanical behavior of subjects may be divided into sports mode hydrostatic and hydrodynamic. Fluid mechanics research is based on Newton's laws of motion and conservation of mass law, often have to use the knowledge of thermodynamics, and sometimes used the basic laws of macroscopic dynamics equations and physics, basic knowledge of chemistry.

Transmission problems, such as blade design fluid machinery, ships and aircraft design, engine design, reaction tank design, and even the semiconductor manufacturing process, such as sputtering or evaporation analysis covers a very wide range of applications. And in addition to this analysis tools adapted to explore the impact of parameter changes, but because of the established analytical database, and more able to reduce the man-hours required for the experiment and shorten design time away. With the progress of the update and the calculation method of computer hardware and software technology, the use of computational fluid dynamics to explore the physical world of the fluid, not only has been widely used by scholars, have become the industry to solve engineering problems related to a major weapon. Today, computational fluid dynamics and theoretical mechanics, fluid dynamics experiments constituted the largest modern mainstream research in fluid mechanics. Has developed a finite difference method, finite element method, finite volume method, spectral method and Sim algorithms, coupled with the vigorous development of computer technology now makes the CPU computational efficiency, memory capacity, storage devices, operating systems and network auxiliary parallel computing, and other peripheral functions under that CFD is widely applied to almost all parts of the field of fluid dynamics.

In the past the process industries often use to drive the rotating disc cylinder or rectangular, thereby to understand the internal structure of the flow field. Applying a simple geometric structure of the flow field to explore rotation can help analyze and understand the principles of early and recent research literature mostly cylindrical or rectangular shape, so this article is to explore the use of a cube shape, in their daily lives the most common is the computer's CDROM drive, using a rotary disc pieces to bring the whole flow field, hoping to apply the same theory to understand the internal structure of the flow field, the paper is a continuation of the literature Chiang [12] study was done to continue to explore when $R e>2000$ the structure of the flow field generated. 
Benjamin and Denny [1] in 1979 with the vorticity-stream function method to simulate the two-dimensional closed pull hole course and supplemented with multi-grid method, the first to raise the numerical simulation of the Reynolds number to 104 , expressly found that the two-dimensional closed pull hole course is located in the geometric center of the main vortex with three corners of the second vortex. Ostrach [3] in 1972 experimentally observed velocity distribution in the cavity exhibits antisymmetric, but the viscosity coefficient is a function of temperature, in a small wall near the thermal boundary layer of fluid, large speed. Fenstermacher [4] in 1979, the use of laser-Doppler velocimetry explore concentric circles in the middle of the restricted fluid from the column of rotating flow generated by the transitional situation. Chenoweth and Paolucci [5] in 1986 as well as Dennis and Hudson [6] in 1989 to study the solid wall boundary movement along the border and around the hole center of rotation.

Goharzadeh and Mutabazi [13] in 2001, the study is to explore is reversed between concentric cylinders, in Couette-Taylor system, the experimental characteristics of intermittent flow conditions. Disturbed flow conditions, the study found that even two-cylinder turbulence in part dependent on the speed with the speed of increase turbulence and then evolved into a spiral turbulence, turbulence generated by its spiral speed when using the control parameters, in order to observe the reverse cylinder axial velocity dependent on the speed of the outside of the column. Okulov, Meledin and Naumov [14] in 2003, when at $R e=1500-6000$ cube rotating disc plus tip vortex experimental studies, when $R e$ is greater than 4000 will have a spiral shape vortex core disintegration will produce steady flow field (unsteady flow) phenomenon. Sparrow and Abraham [15] mentioned assumptions virtual density in 2003, will lead to numerical errors, so the momentum equation calculations, based on the direct method for solving the momentum Fang Cheng style. And referred to the closed cavity natural convection, fluid flow is not only caused by the density difference, we must consider the impact of pressure on the flow field. Doby, Nowakowski and Dyakowski [16] in 2007 to study for a cylindrical vortex flow generated by adding a rotating cover using experimental and numerical methods.

This paper is divided into five chapters, are outlined as follows: Section 2 for the physical and mathematical model, a brief governing equations used herein, the governing equation is substituted into the hypothetical dimensionless parameters, push dimensionless equations, assuming that the boundary conditions. In section 3 , the numerical square model, numerical methods used in this paper is to keep the format of the dispersion relation to reduce the error of the convective terms, and then using the finite difference method to illustrate the problem with the program verification. Section 4, the numerical results are plotted in figure flow field and for the impact caused by the different number Re discuss research. Finally section, the comparing the results obtained for the post and then make a summary statement of results and the contribution of this chapter.

\section{NUMERICAL MODEL}

This article assumes that the entire flow field for incompressible flow (incompressible flow), all are all threedimensional flow field flow field, Figure 1, the top has a rotating disk, the disk radius equal to feature here length $L$, maximum rotational speed of the disc is $(R \Omega)$ equal to the characteristic velocity $u_{\infty}$. The use of three-dimensional Navier-Stokes equations transient Cartesian coordinate system, the three-dimensional transient continuous equation, momentum equation and energy equation is simplified as follows:

$$
\begin{aligned}
& \frac{\partial u_{i}}{\partial x_{i}}=0 \\
& \frac{\partial u_{i}}{\partial t}+\frac{\partial}{\partial x_{m}}\left(u_{m} u_{i}\right)=-\frac{\partial p}{\partial x_{i}}+\frac{1}{R e} \frac{\partial^{2} u_{i}}{\partial x_{m} \partial x_{m}}
\end{aligned}
$$

The above two equations will be solved subject to the initial divergence-free velocity condition and boundary velocities to close the problem. In this primitive-variable formulation, specification of velocity boundary conditions has been rigorously proven.

A small and smooth change of the parameter value (or $R e$ in the currently investigated differential system) may cause a sudden qualitative or topological change in the system equilibrium. Under the circumstances, we call that bifurcation initiates. According to the theory of bifurcation, which is in association with the local solution behaviors of nonlinear equation in the neighbor of a known solution of the equation, bifurcations can be divided into the local and global types. Local bifurcations are mathematically associated with the real part of the eigenvalues of an equilibrium which passes through zero. Local bifurcation, which includes pitchfork, Hopf, saddle-node (or tangent or blue sky), periodic-doubling (flip), secondary Hopf (Neimark), and transcritical bifurcations, occurs when the Reynolds number change causes the stability of an equilibrium to change. Since only the local bifurcation type has been found in this study in the present nonlinear system, no global bifurcation will be dealt with herein.

\subsection{Dispersion-Relation-Preserving (DRP) Scheme}

Finite difference method (FDM) is a method used to calculate the numerical simulation of the oldest, is still widely used. Partial differential equations to be solved, by Taylor expansion, the direct control of discrete equations, which are covered by the concept of localized discrete. For the finite difference scheme, from precision format to points, there is a first-order scheme, second order and higher-order format. From space to consider the differential form, can be divided into the center format and upwind format. Consider the impact of the time factor; the difference scheme can also be divided into explicit form, implicit schemes, explicit and implicit alternate format. Current common difference scheme, mainly in the form of a combination of the several different combinations constitute 
different differential formats. There are many ways constitute a difference, now mainly used in the Taylor series expansion method. The basic differential expression of three major forms: first-order forward difference, first-order backward difference, first-order and second-order central difference central difference, of which the first two formats for the first-order accuracy, the latter two formats for the second-order calculation accuracy. The main disadvantage of the finite difference method is poor and the applicability of the numerical solution of complex conservation area is difficult to ensure, so this part of the diffusion terms in the discrete second order accurate difference method middle.

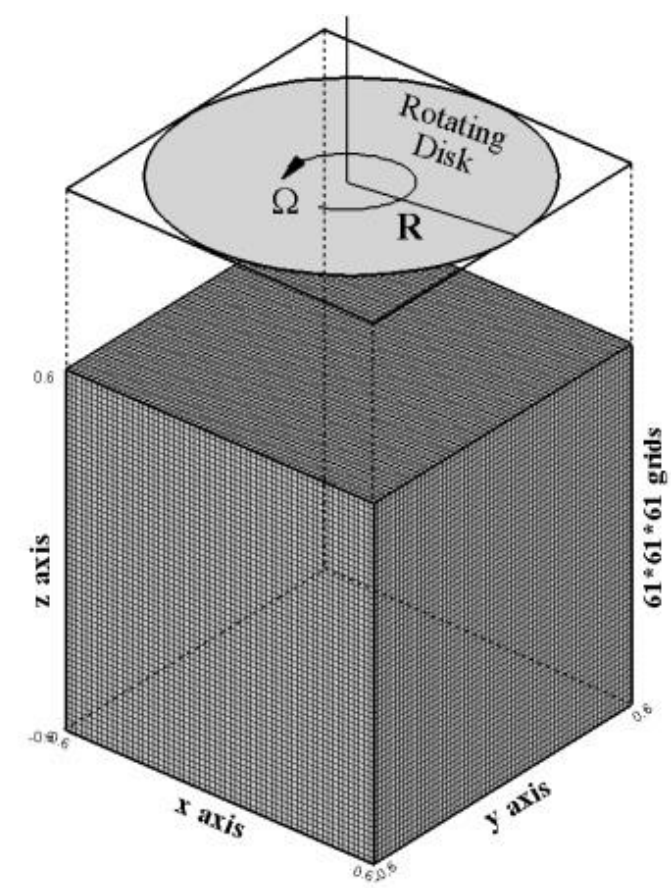

Fig-1: Description of the investigated natural-convection problem and the uniform grid distribution on the bounding surfaces.

In the past literature, by Tam and Webb put forward dispersion relations have maintained format (dispersionrelation-preserving scheme, hereinafter referred to as DRP format), use of best spatial discrete methods to increase the number of analog format on the wave power. The idea is to assume the function of a Fourier transformation, then the Fourier converted. The corresponding difference scheme inevitably transformed with the presence of poor guide. DRP format while maintaining a certain accuracy of the premise, to make such a poor dispersion coefficient optimization guide smallest, reduce errors convection term.

\subsection{Total Variation Diminishing (TVD) Scheme}

Total variation diminishing (TVD) scheme is proposed by Harten et al., TVD format is a semi-discrete scheme up to reach the second-order accurate precision. But the critical point will be reduced to first-order accuracy, and therefore Harten and Osher et al ENO (Essentially Non-Oscillatory) format, thereby improving the accuracy and characteristics of TVD scheme does not oscillate, so that in any case TVD format maintain high order accuracy.
Generally when solving ordinary differential equations, according to the accuracy required to select the correlation coefficient for a typical fourth-order accurate Runge-Kutta explicit time integration count is concerned, then select $\alpha_{1}=\alpha_{2}=0.5, \alpha_{3}=1, \beta_{0}=\beta_{3}=1 / 6$ and $\beta_{1}=\beta_{2}=1 / 3$ 。 Runge-Kutta count the count is compared with Euler, although they may be bigger, but not necessarily to calculate the total amount of savings, because solving the main computational calculations, four times longer to be calculated at each time step $\Delta t$, but the opposite can't is increased 4 times. Runge-Kutta currently considered are mainly used for high accuracy requirements on the issue.

\section{NUMERICAL RESULTS}

This is the top of the cube plus rotating disk drive, and to explore the structure of the flow field, the continuation of the literature Chiang [12] have done research, more in-depth discussion the case when the Reynolds number flow field to increase the structural change, and learn about their physical phenomenon occurs. The numerical results obtained in this article essays Fortran programs found at the top of the rotation when $R e>4800$ will produce unstable flow (unsteady), both up and down the rotation is $R e$ will produce 4000 , so this article is divided into three parts to investigate, the first part is a steady flow (steady) $R e=500$, 2000, 2500, 3000, 3500 and 4000 taken for analysis. The second part is the unstable flow, its velocity, pressure, flow structure will change over time, so this takes time to analyze $R e=7000$. The last part of the rotation of the upper and lower case, $R e=5000$ to take can take to analyze for comparison with the second part.

\subsection{Steady Flow Structural Analysis Field}

This paper discusses the first to enter, the talk in the past of literature than to be right, to learn about the applicability and accuracy of the numerical Figure 1 Chiang literature of comparison, the figure can be learned from a slight gap between the reason may be is the number of different grid use, so different numerical methods used so causing a slight error. Figure 2 is compared with the results of experiments done right, can be learned from the figure when $R e=2000$, little error at $R e=4000$, then it is open to explore entering this article. 


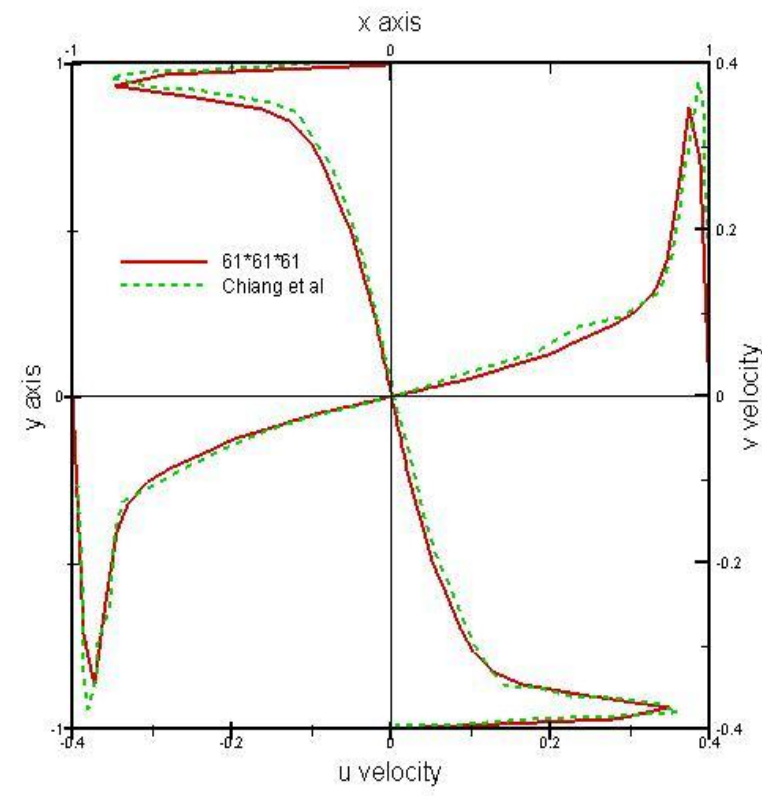

(a)

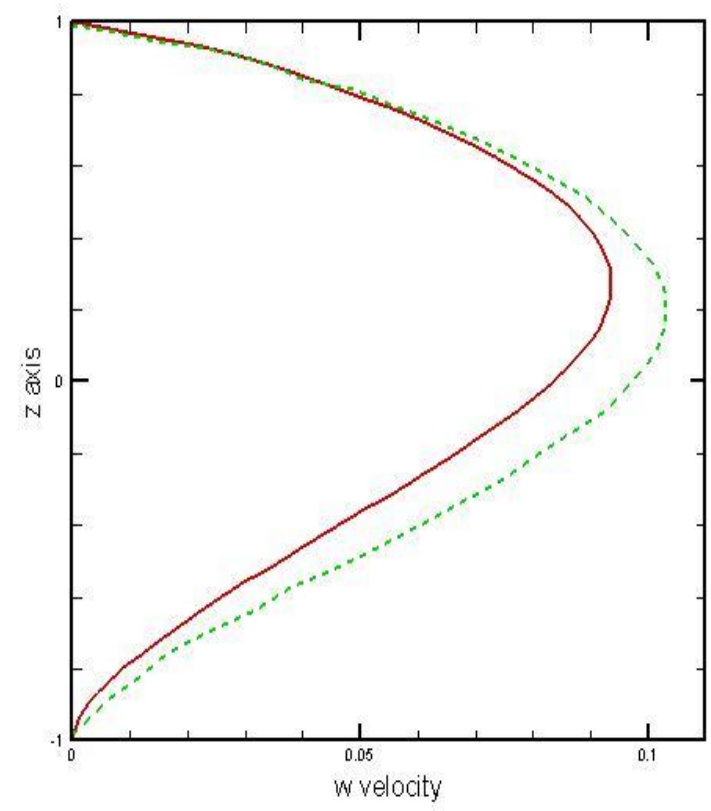

(b)

Fig-2: When $\mathrm{Re}=1000$, the numerical results with the literature than (a) $u(0, y, 0)$ and $v(x, 0,0)$; (b) $w(0,0, z)$.

Figure 3 Comparison of the speed in the $z$ direction, from the figure that as the Reynolds number can increase the speed will be higher. Next, in Figure 4 from the plane $y=0$ for four different Reynolds numbers flow field pattern, it can be observed from the figure had a different rotational direction of the vortex and the center of the vortex near the top of the cavity, this is because the top a rotating disk ( $z$ direction is the angular velocity), prompting the fluid passes through the center of the chamber to flow upward, fluid particles flowing near the cavity direction downwardly into the vertical end wall, thereby forming a secondary flow structure is inverted. Different from Figure 5(a) $z$ direction of the flow field pattern surface can be observed that all the flow is from the boundary wall to the middle of the stream into the flow field around the structure plus the fluid rotating in part caused by the middle of the inflow biggest reason distorted, $z=0.4$ in the four corners of the surface can be observed that there are four distinct secondary flow, because the relationship between the inverse pressure gradient caused by the occurrence of reflux, the formation of secondary flow in the four corners of the since viscous shear stress of the impact caused. From the vorticity 5(b) observed $z$ direction, this flow field is a rotating flow it will generate vorticity, vorticity can be seen from the vorticity in the middle part of the largest, it is because the amount of rotation in the middle with both shear stress so large relative vorticity is relatively large near the smaller boundary layer vorticity, and the greater the surface vorticity near the top of the disc.

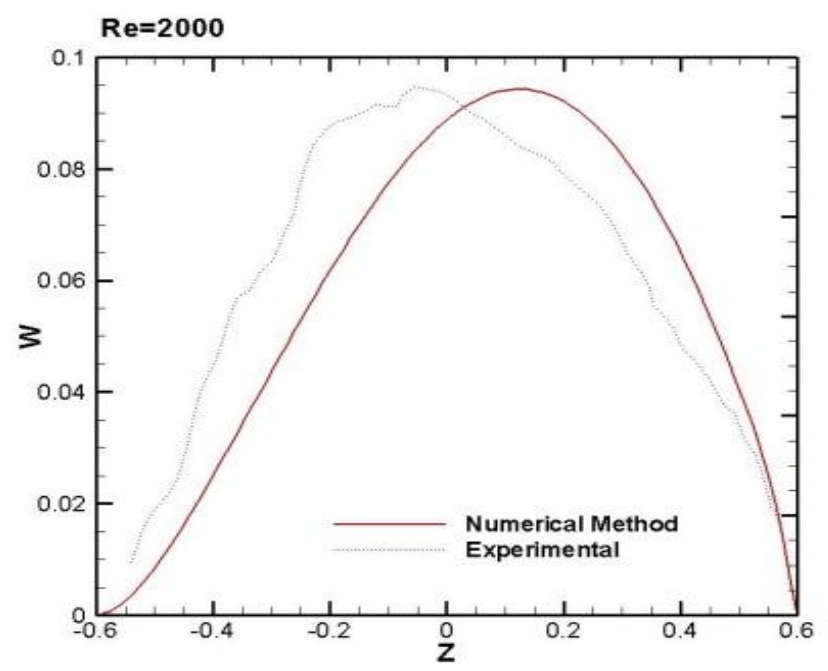

(a)

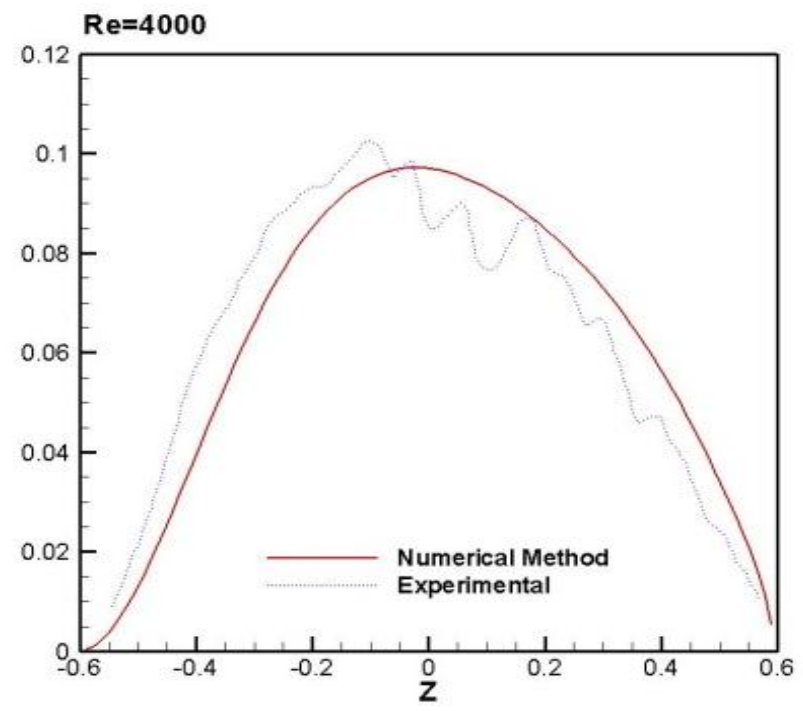

(b)

Fig-3: With the experimental results in the literature than (a) $\operatorname{Re}=2000$; (b) $\operatorname{Re}=4000$. 


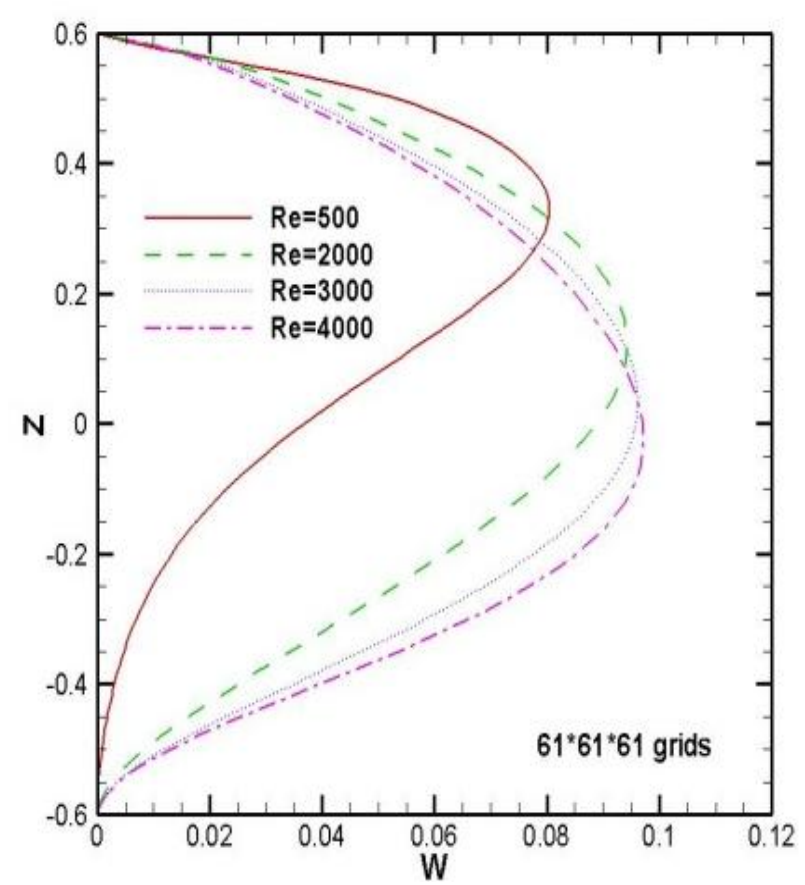

Fig-4: Compare $R e$ at the same grid

From 5(c) pressure chart with 5(d) the speed chart, can be observed in the middle of the maximum speed of the smaller and more close to the border, because the border is no slip boundary condition (no-slip) minimum speed so close to the border, but because so when will flow into the closer contact with the boundary layer the greater the pressure boundary. And from the speed can also explain why in the middle of the main vortex vorticity will be greater than four secondary flow vorticity. The use of topology theory can be obtained by flow into a spiral point (attracting-spiral 2D), at $y= \pm 0.6$, $x= \pm 0.6, z=-0.6$ and $z=0.6$ has two faces, plotted as a threedimensional map Figure 6(a) and 6(b), from the figure can be found at the top of a counter-clockwise rotation of the disk can be used to drive the flow field, from each of the boundary wall flows into the direction of flow can be learned, when $R e=500$ time the entire flow field flow direction is along the direction of rotation of the disc flow.

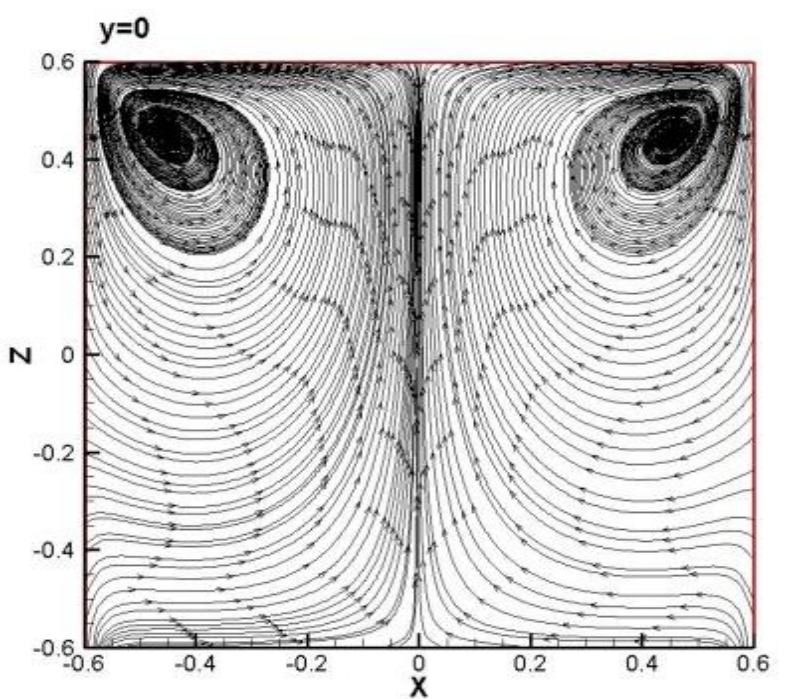

(a)

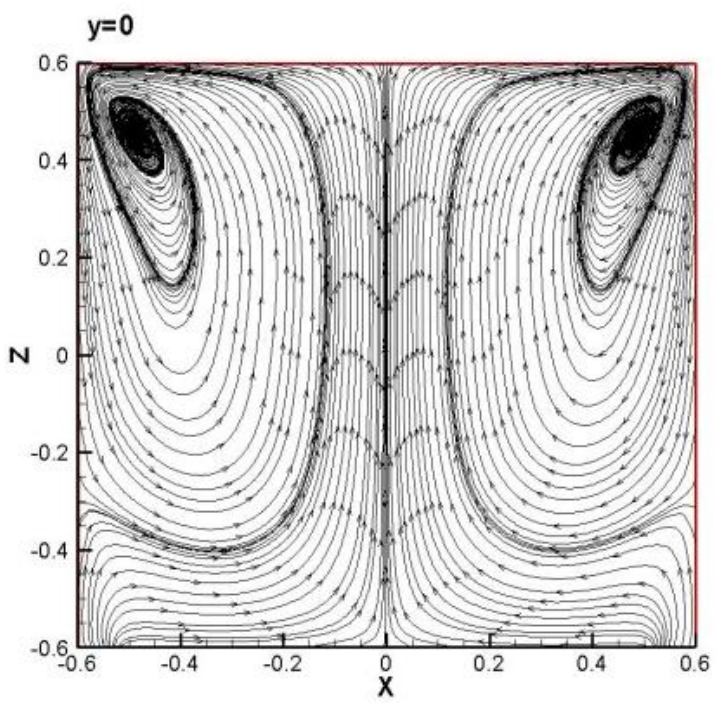

(b)

Fig-5: At different $\mathrm{Re}, \mathrm{y}=0$ plane flow field pattern (a) $\operatorname{Re}=500$; (b) $\operatorname{Re}=2000$.
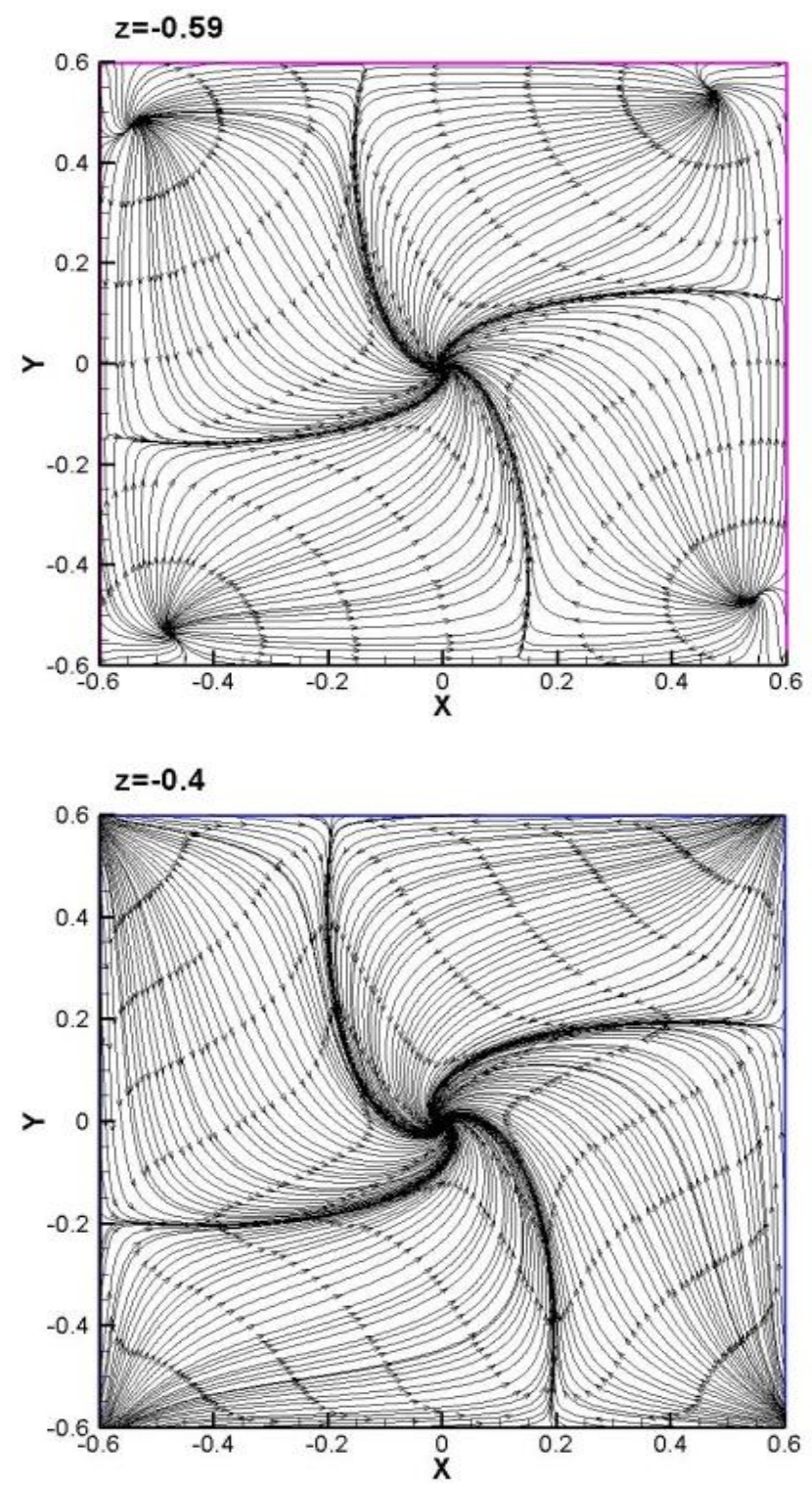

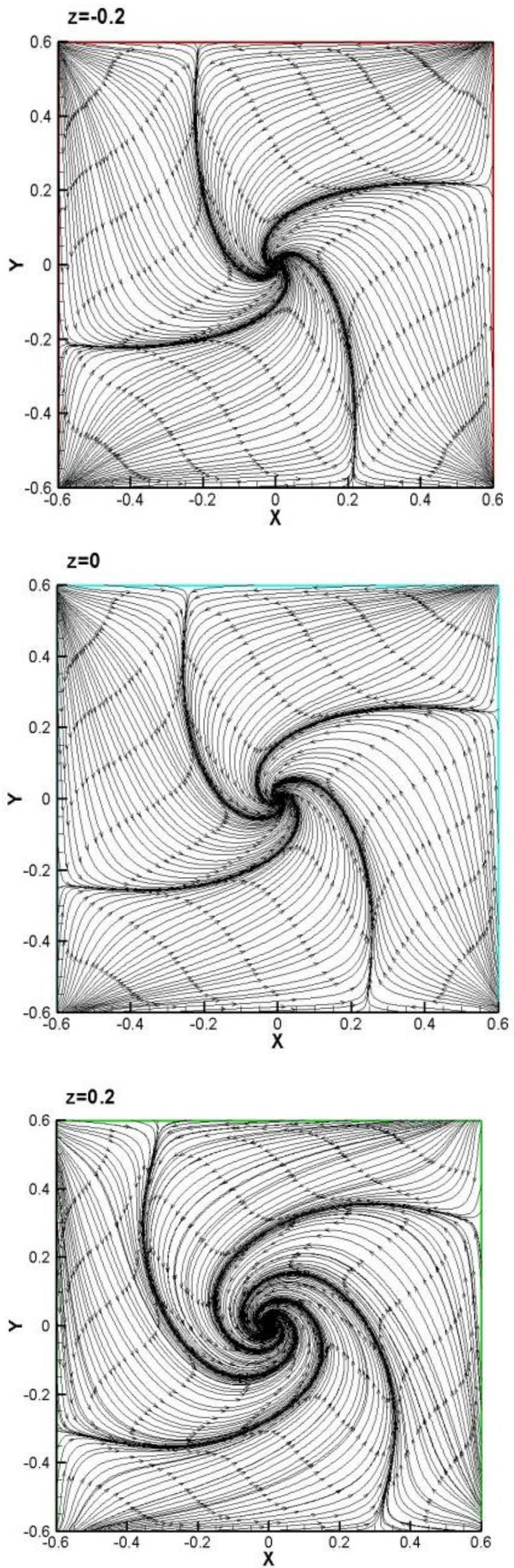

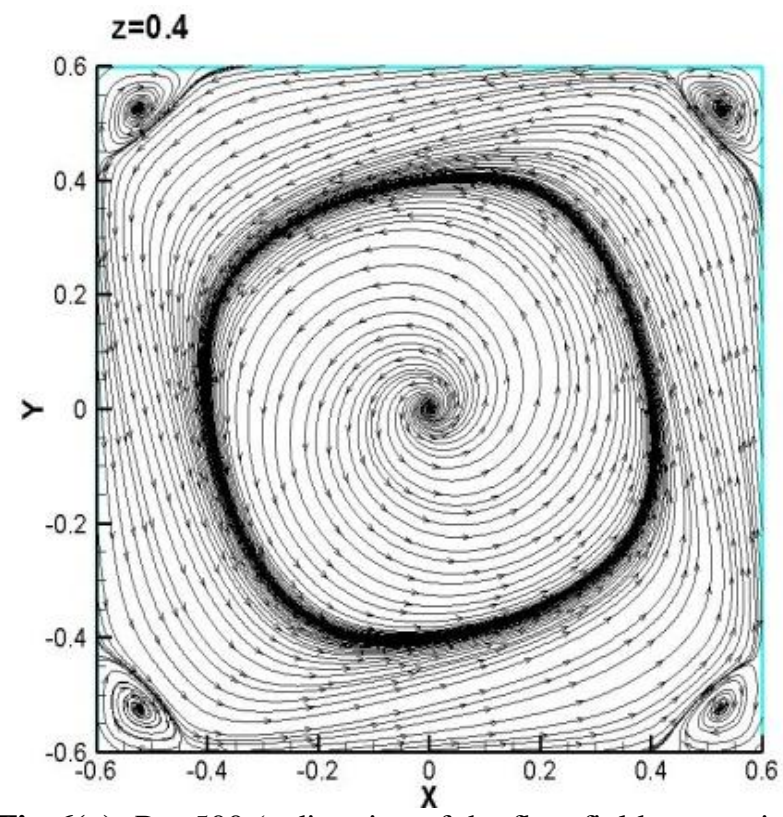

Fig-6(a): $\mathrm{Re}=500$ ( $\mathrm{z}$ direction of the flow field pattern in a different plane)

\subsection{Unsteady Flow Field Structure Analysis}

From the program can be learned when $R e>4800$ will produce non-stable (unsteady) phenomenon during, due to unsteady flow field will change over time, so this takes $R e=7000$ to analyze the convergence of the cycle can be observed when the time $\mathrm{t}=500$ then will produce peaks, due to find a regular cycle from peak time to take $t=500 \sim 525$, every one second to take group data.

Because it will change over time so this taking $t=501 \sim 503$ to the tip of these three analyzes, from Fig. 6 speed and 7 pressure comparison chart, can clearly observe the pressure with speed will change over time. From Figure 4-27 (a) with Figure 8 pressure with speed also change over time. From Figure 8 it is clear that over time, the flow structure changed significantly different, and the Topology theory calculated to produce two break points at each time will be.

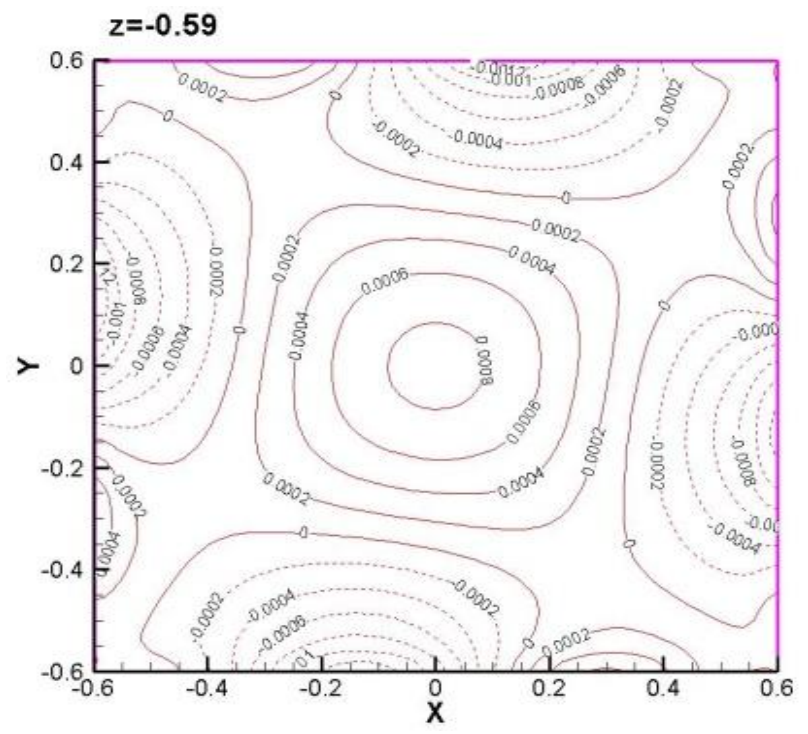



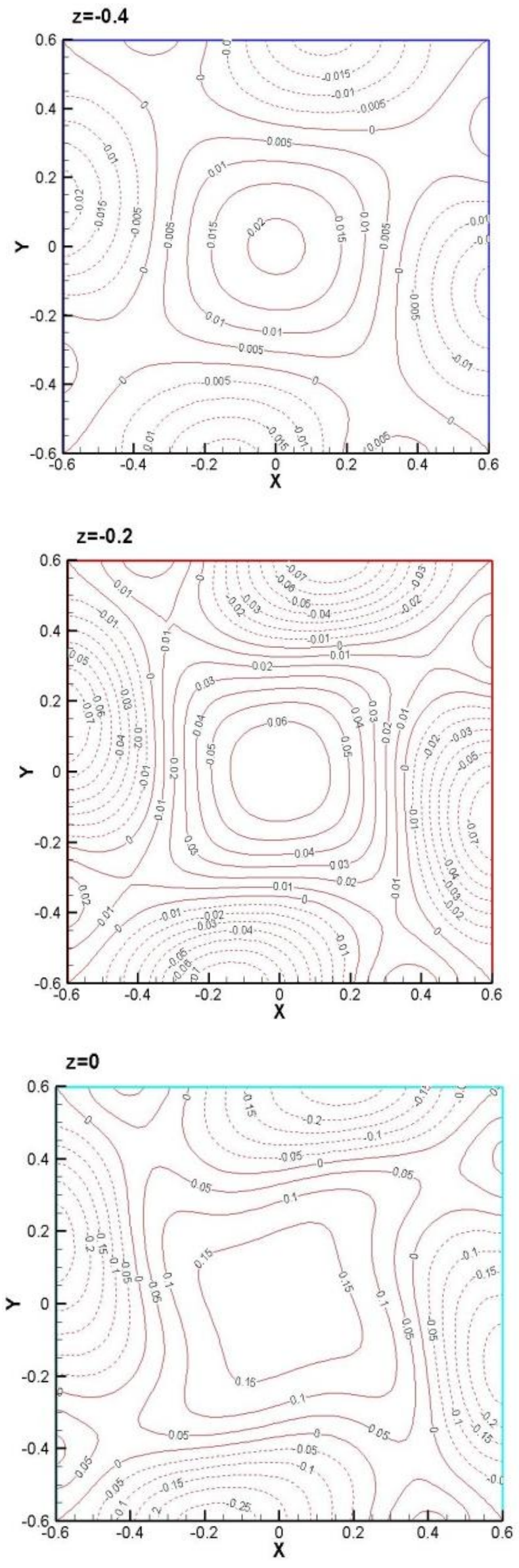
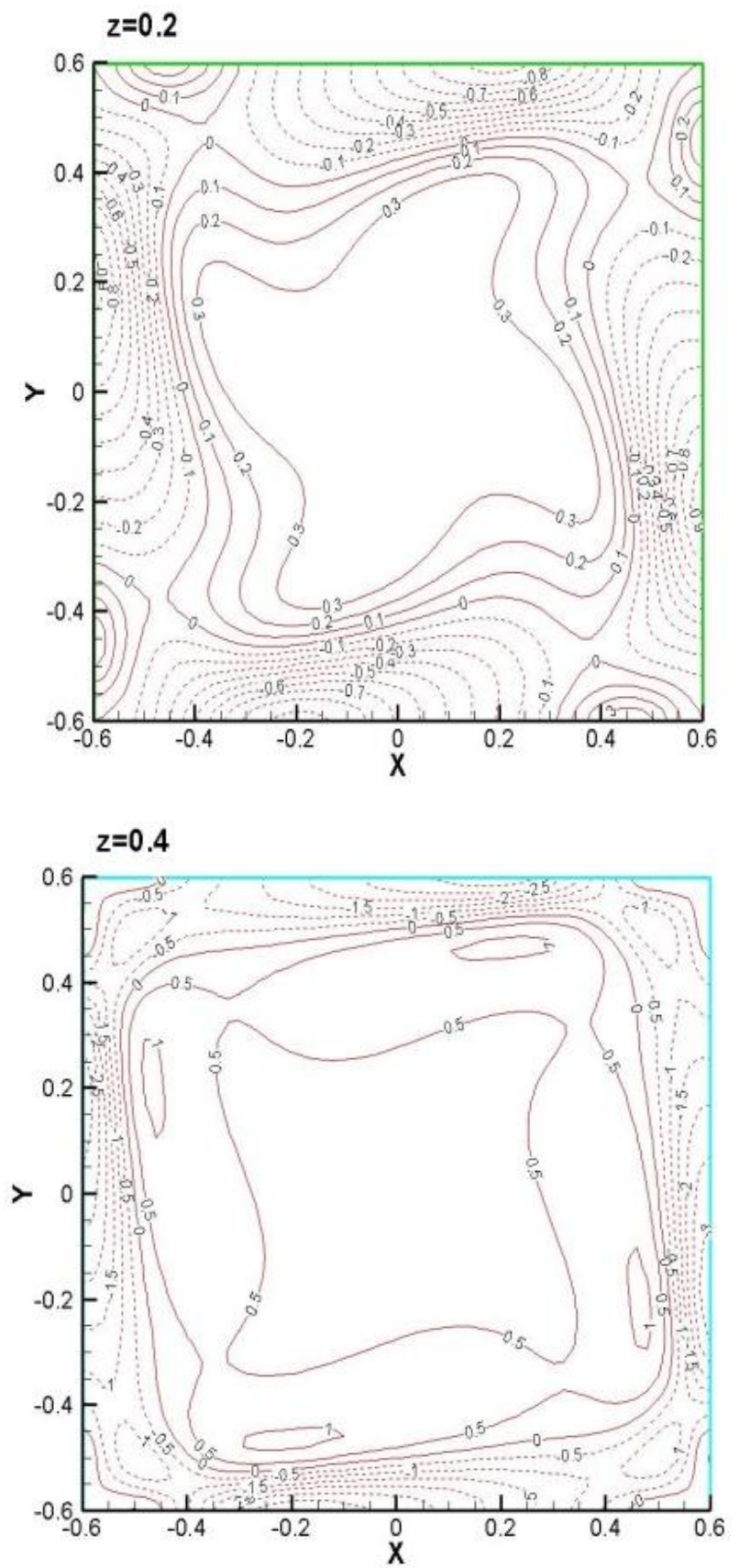

Fig-6(b): $R e=500$ ( $z$ direction vorticity map in a different plane)

\section{CONCLUSION}

The present study is to investigate the flow field at different Reynolds numbers, mainly to explore when $R e=500,2000$, 3000, 4000, 7000 also have to add a disk drive down $R e=5000$ case to make research and analysis, by Numerical results into a steady flow structure, unsteady flow field structure of these two parts, $R e$ steady flow structure under study range from 500 to 4000 , unsteady flow field is taken down $R e=7000$ and have disk drives $\mathrm{Re}=5000$ to make analysis, analysis of its singularity characteristics of each surface to calculate the flow into the spiral point, draw a three-dimensional flow field pattern in order to understand the situation led to the use of the entire flow field Topology theory. 
At $R e=500 \sim 4000$ analysis found that the flow of the flow field is very similar, except that as the Reynolds number increases speed and pressure will change, because the speed of the flow field flow lines caused by change-intensive, but the basic structure of the flow field which no significant change in flow pattern. Topology calculated singularity theory use, can be found along with the increase of Reynolds number flow into a spiral point position will gradually near the bottom of the cavity, and the Reynolds number increases as the impact of a singular point in the bottom of the significantly smaller. Surface 4 at $z=-0.59$ vortex sustained close to the end wall, this is because as the Reynolds number increases speed to follow the change quickly, so when the speed is increased stiction force is relatively minor role it will go boundary near the end wall. By unsteady flow field structure analysis types can be learned, in the case of high Reynolds number can cause cracking point, thus changing the structure of the entire flow field.
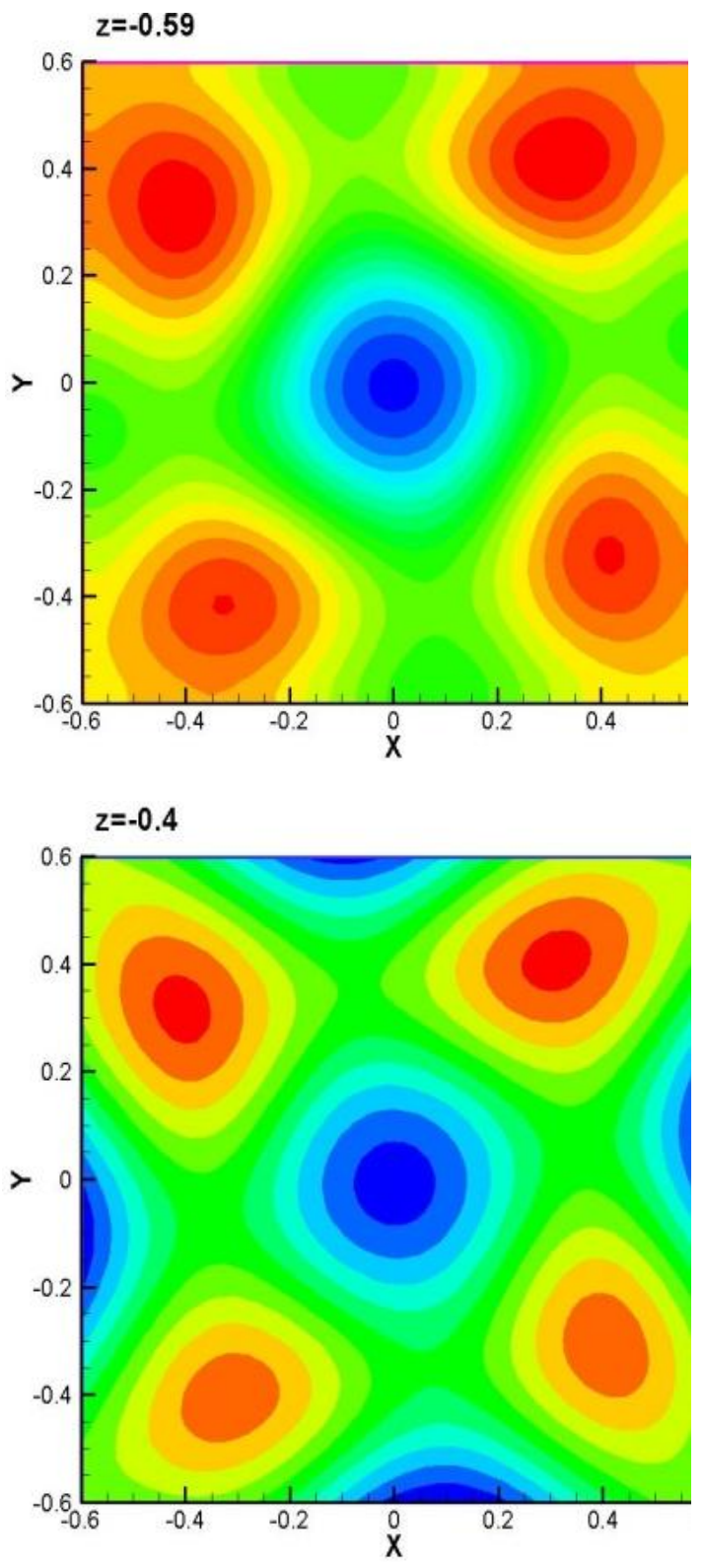
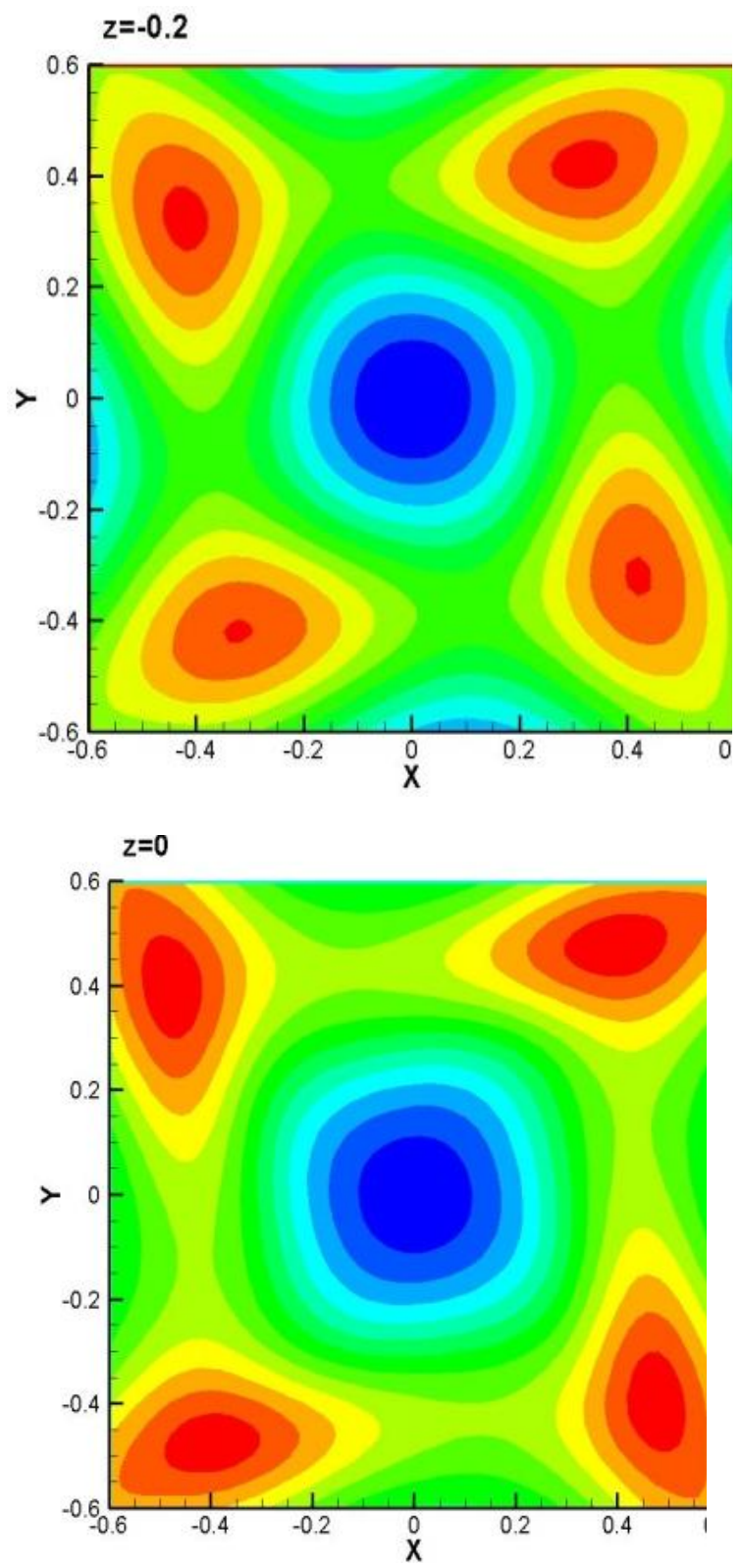

$z=0.2$

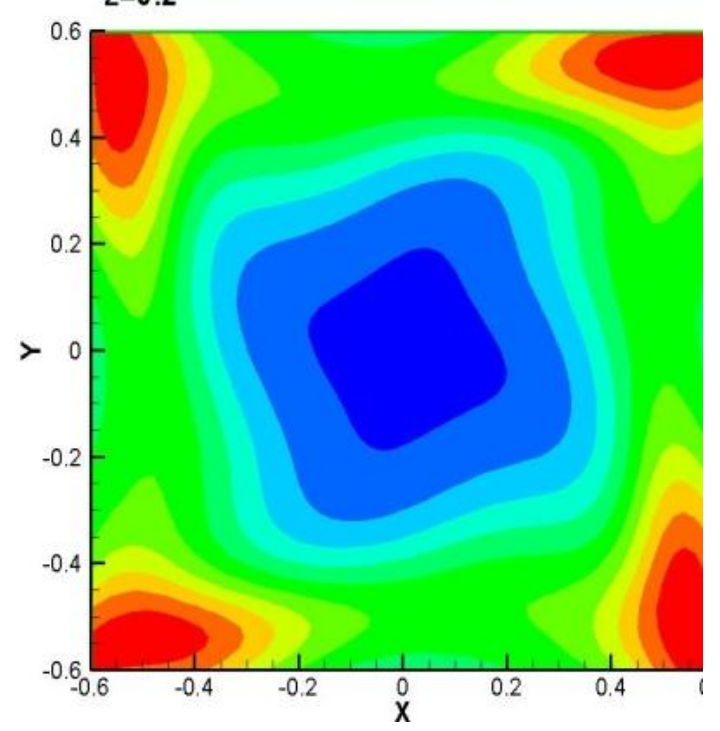




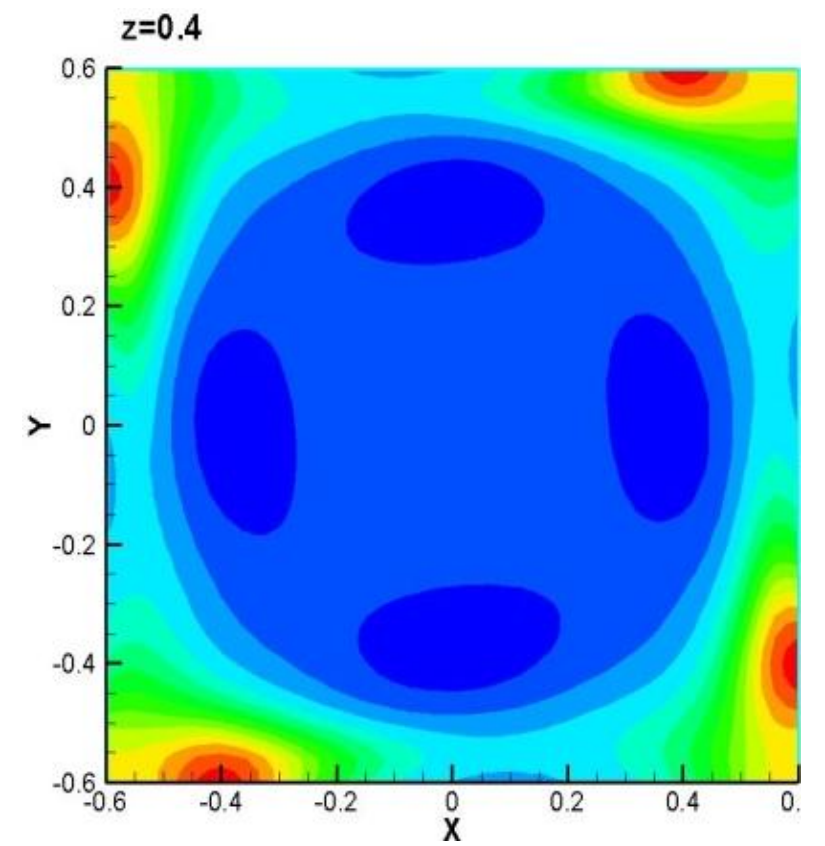

Fig-6(c): $R e=500$ ( $z$ direction pressure diagram on different faces)

\section{ACKNOWLEDGEMENTS}

The financial supports provided by the Ministry of Science and Technology (MOST) under grants MOST 102-2221-E229-001 and MOST102-2119-M-492-004 are gratefully acknowledged.

\section{REFERENCES}

[1] T. P. Chiang, W. H. Sheu, S. F. Tsai, Disk-driven vortical flow structure in a cubical container, Computers \& Fluid., Vol. 28 : 41-61(1999).

[2] Christopher K. W. Tam, Jay C. Webb, "Dispersionrelation-preserving finite difference schemes for computational acoustics, J. Comput. Phy., Vol. 107 : 262-281(1993).

[3] Harten . A, Osher. S, Chakravarthy S. R. "Uniformly high-order accurate essentially nonoscillatory schemes 3"J. Comput. Phys., Vol. 71 : 231-303(1987)

[4] A. S. Benjamin, V. E. Denny, On the convergence of numerical solution for 2-D flows in a cavity at large Re, J. Comput. Phys., Vol. 33 : 340358(1979).

[5] Takaji Inamuro, Akimasa Yamaguchil and Fumimaru Ogino, Fluid flow in a rotating cylindrical container with a rotating disk at the fluid surface, Fluid Dynamics Research., Vol. 21 : 417430(1997).

[6] V. L. Okulov, V. G. Meledin, and I. V. Naumov, Experimental Investigation of a Swirling Flow in a Cubic Container, Technical Physics., Vol. 48 : 1249-1254(2003)

[7] A Harten, S Osher, SR Chakravarthy (1987) Uniformly high-order accurate essentially non- oscillatory schemes. J. Comput Phys, Vol. 71: 231303

[8] GD Thiart (1990) Finite difference scheme for the numerical solution of fluid flow and heat transfer problems on non-staggered grids, Numer Heat Tran, Part B, Vol. 17: 43-62

[9] T Fusegi, JM Hyun, K Kuwahara, B Farouk (1991) A numerical study of three-dimensional natural convection in a differentially heated cubical enclosure, Int. J. Heat Mass Transfer Vol. 34: 15431557

[10] Christopher KW Tam, Jay C Webb (1993), Dispersion-relation-preserving finite difference schemes for computational acoustics, J. Comput. Phys, Vol. 107: 262-281

[11] TL Lee, TF Lin (1996) Transient three-dimensional convection of air in a differentially heated rotating cubic cavity, Int. J. Heat Mass Transfer Vol. 39: 1243-1255

[12] YT Ker, TF Lin (1996) A combined numerical and experimental study of air convection in a differentially heated rotating cubic cavity, Int. J. Heat Mass Transfer, Vol. 39: 3193-3210

[13] W Date (1993) Solution of Navier-Stokes equations on non-staggered grids, Int. J. Heat and Mass Transfer, Vol. 36: 1913-1922

[14] Shinichiro Wakashima, Takeo S Saitoh (2004) Benchmark solutions for natural convection in a cubic cavity using the high-order time-space method, Int. J. Heat \& Mass Tran, Vol. 47: 853-864

[15] C. Kuhlmann, M. Wanschura, H. J. Rath, Flow in two-sided lid-driven cavity: Non-uniqueness, instabilities, and cellular structures. J. Fluid Mech., 336, 267-299, 1997

[16] S. V. Patanker, Numerical Heat Transfer and Fluid Flow. Hemisphere, 1980

[17] A. Ladyzhenskaya, Mathematical Problems in the Dynamics of a Viscous Incompressible Flow. Gordon and Breach, New York, 1963

[18] S. Abdallah, Numerical solution for the incompressible Navier-Stokes equations in primitive variables using a non-satggered grid, II. J. Comput. Phys. 70, 193-202, 1987

[19] P. H. Chiu, T. W. H. Sheu, R. K. Lin, Development of a dispersion-relation-preserving upwinding scheme for incompressible Navier-Stokes equations on non-staggered grids. Numer. Heat Tran. Part B, 48(6), 543-569, 2005

[20] L. Quartapelle, M. Napolitano, Integral condtions for the pressure in the computation of incompressible viscous flows. J. Comput. Phys. 62, 340-348, 1986

[21] P. Lin, A sequential regularization method for timedependent incompressible Navier-Stokes equations. SIAM J. Numer. Anal. 34(3), 1051-1071, 1997 

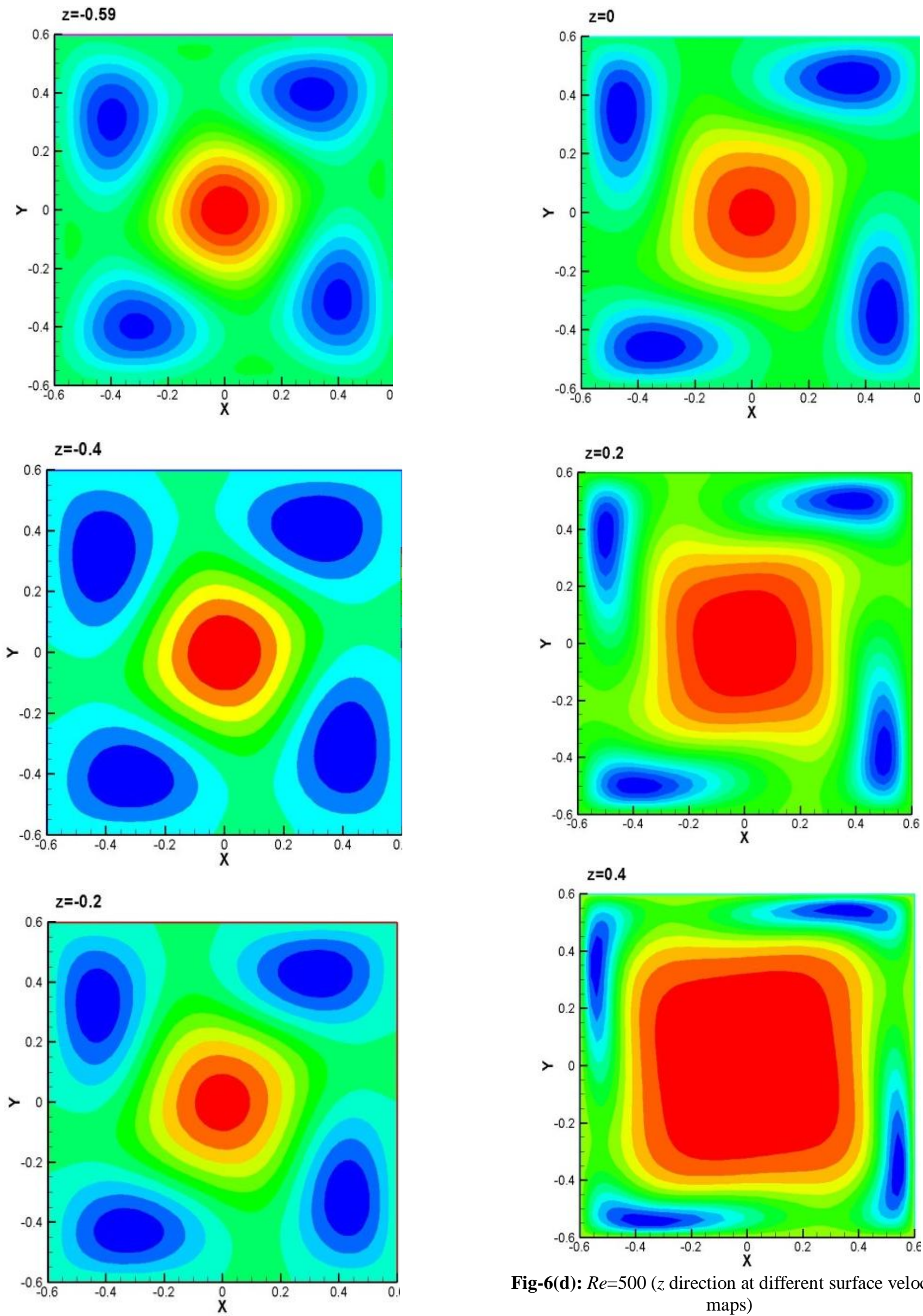

Fig-6(d): $R e=500(z$ direction at different surface velocity maps) 


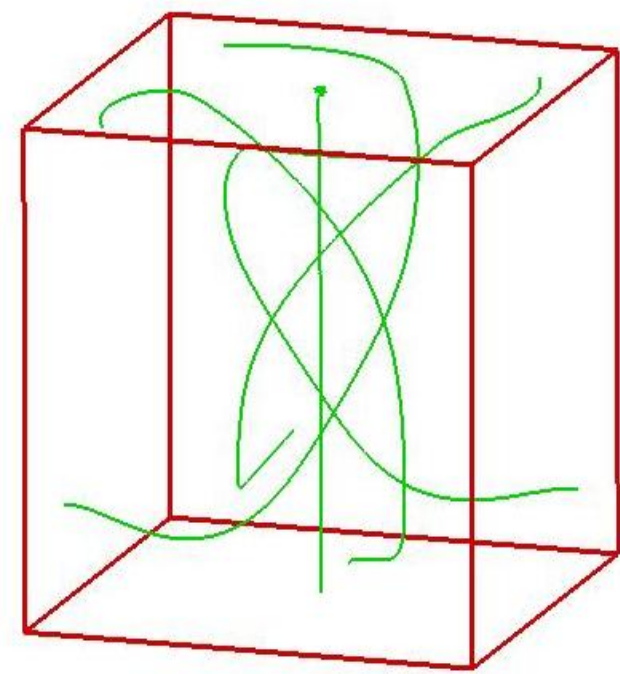

(a)

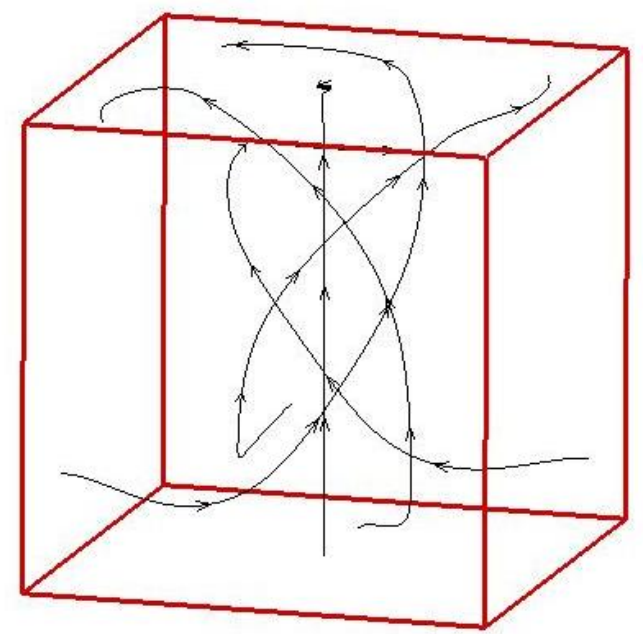

(b)

Fig-7: $R e=4000$ vortex core flow field pattern

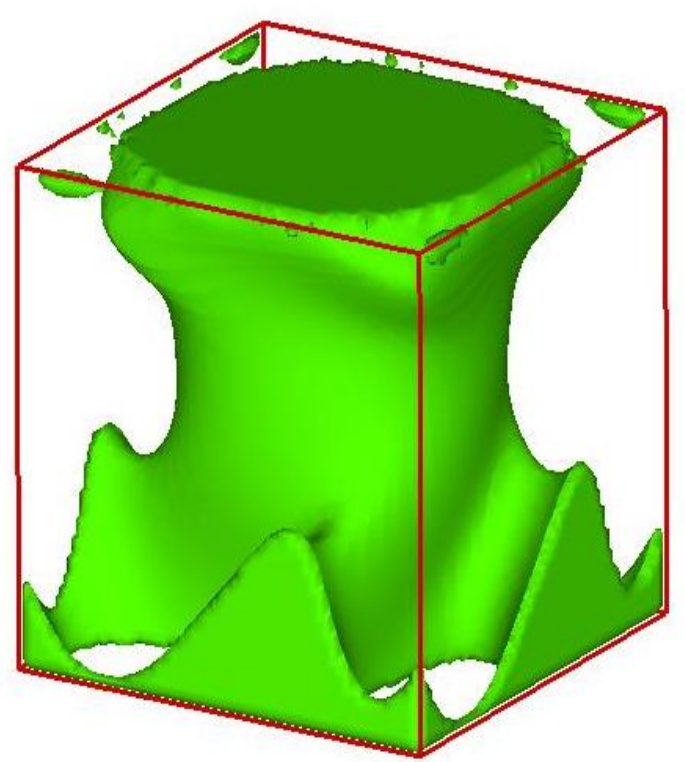

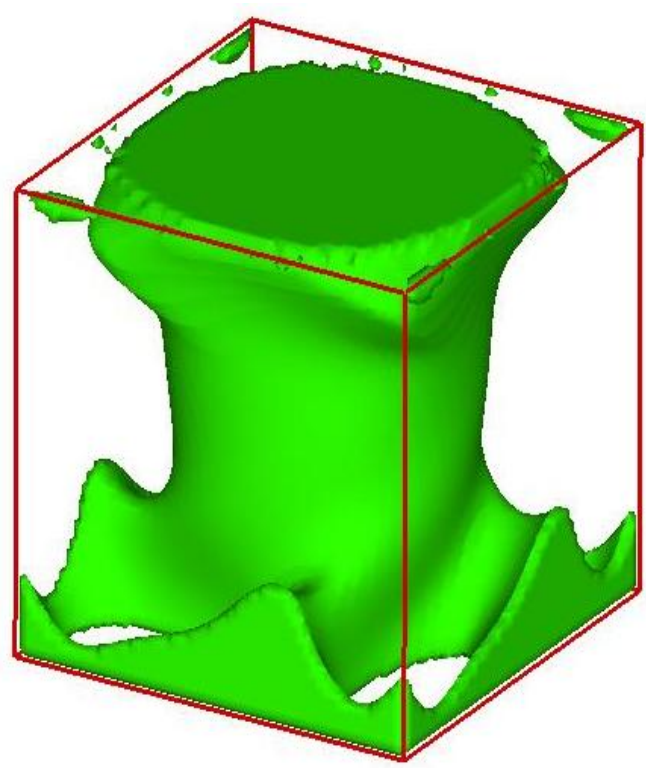

(b)

Fig-8: $w=0$ in different iso-surface $R e$ : (a) $R e=2000$; (b) $R e=3000 ;(\mathrm{c}) \operatorname{Re}=4000$

\section{BIOGRAPHIES}

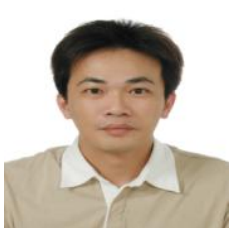

Reui-Kuo Lin is assistant professor of Marine Engineering, Taipei College of Maritime Technology, Taipei, Taiwan, ROC. His PhD from the National Taiwan University, Taiwan, ROC at 2005. His domain of interest is: computational fluid dynamics, two-phase flows and applied mathematics.

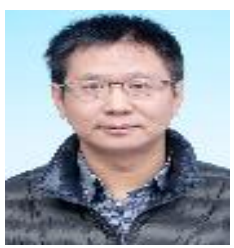

Kwan Ouyang is associate professor of Marine Engineering, Taipei College of Maritime Technology, Taipei, Taiwan, ROC. He obtained his PhD from the Chung Cheng Institute of Technology, National Defense University, Taiwan, ROC at 2008. His domain of interest is: computational fluid dynamics and drag reduction.

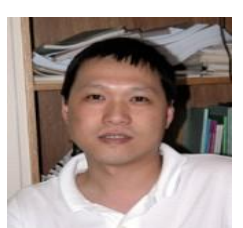

Sheu-Feng Tsai is a Assistant Professor of Marine Engineering, National Taiwan Ocean University, Taiwan, ROC. He obtained his $\mathrm{PhD}$ from the National Taiwan University, Taiwan, ROC at 2001. His domain of interest is: Computational Fluid Dynamics, Heat Transfer, Hemodynamics, and Biomechanics.

(a) 